Commun. math. Phys. 28, 245-249 (1972)

(C) by Springer-Verlag 1972

\title{
An Entropy Inequality for Quantum Measurements
}

\author{
G. Lindblad
}

Department of Theoretical Physics, Royal Institute of Technology, Stockholm, Sweden

Received February 24, 1972; in revised form June 12, 1972

Abstract. It is proved that for an ideal quantum measurement the average entropy of the reduced states after the measurement is not greater than the entropy of the original state.

Consider a quantum state described by a density operator $W$ in Hilbert space

$$
W=W^{*} \geqq 0, \quad \operatorname{Tr} W=1 .
$$

Then there exists an orthonormal set $\{|i\rangle\}$ such that

$$
W=\Sigma w_{i} W_{i}
$$

where $W_{i}=|i\rangle\langle i|, w_{i}>0, \Sigma w_{i}=1$.

Let $O$ be an observable with eigenspaces defined by projections $P_{k}$;

$$
O=\Sigma \omega_{k} P_{k}, \quad \Sigma P_{k}=I .
$$

If $O$ is measured the value $\omega_{k}$ is obtained with probability $p_{k}=\operatorname{Tr} W P_{k}$ and $W$ is then replaced by

$$
W_{k}^{\prime}=p_{k}^{-1} P_{k} W P_{k}
$$

The average over all possible outcomes gives a density operator

$$
W^{\prime}=\Sigma P_{k} W P_{k}=\Sigma p_{k} W_{k}^{\prime}
$$

(sum over all $k$ such that $p_{k} \neq 0$ ).

The entropy of a state $W$ is defined as

$$
S(W)=-\operatorname{Tr} W \log W=-\Sigma w_{i} \log w_{i} .
$$

It is well known that $S(W) \leqq S\left(W^{\prime}\right)$ [1] (the transformation $W \rightarrow W^{\prime}$ is "dissipative") with equality if and only if $W=W^{\prime}$. [A simple proof: use $\operatorname{Tr} W^{\prime} \log W^{\prime}=\operatorname{Tr} W \log W^{\prime}$ and Klein's inequality $\operatorname{Tr}\left(W \log W-W \log W^{\prime}\right)$ $\geqq 0$ ([2], p. 27).]

It was conjectured by Groenewold [3] that the average of the entropies of the states $W_{k}^{\prime}$ is not larger than $S(W)$ :

$$
S(W)-\Sigma p_{k} S\left(W_{k}^{\prime}\right) \geqq 0 \text {. }
$$


If entropy is identified with "missing information" the difference may be interpreted as the average information gain in the measurement process.

It is the object of this paper to prove this inequality (Theorem 2). The proof uses a subadditivity property of the entropy which is proved in Theorem 1.

Theorem 1. Let $A, B$ be positive operators of trace class and let

$$
\begin{aligned}
S(A) & =-\operatorname{Tr} A \log A \text { if } A \log A \text { is of trace class } \\
& =\infty \text { otherwise. }
\end{aligned}
$$

Then $S(A+B) \leqq S(A)+S(B)$.

If $S(A)$ or $S(B)=\infty$ then $S(A+B)=\infty$. If $S(A)$ and $S(B)$ are finite then the equality holds if and only if $A \cdot B=0$.

A proof of the inequality (formulated differently) has been given by Lanford and Robinson [4]. We give an elementary proof which also gives the condition for equality.

Lemma. If $A, B$ are positive trace class operators such that $S(A)<\infty$ and $A \leqq B$ then

$$
\operatorname{Tr}(A \log A-A \log B) \leqq 0
$$

with equality iff $B \mid$ Range $A=A \mid$ Range $A$.

Proof. Let $\left\{\left|a_{i}\right\rangle\right\},\left\{\left|b_{j}\right\rangle\right\}$ be complete orthonormal sets of eigenvectors for $A$ and $B$. We first show that $\operatorname{Tr}\left(B^{-1} A^{2}\right) \leqq \operatorname{Tr} A$.

$A \leqq B$ implies that $A+\varepsilon I \leqq B+\varepsilon I$ for every $\varepsilon>0$. Then $A+\varepsilon I$ and $B+\varepsilon I$ have bounded inverses and ([2], p. 28)

$$
(B+\varepsilon I)^{-1} \leqq(A+\varepsilon I)^{-1} .
$$

Hence $\left\langle a_{i}\left|(A+\varepsilon I)(B+\varepsilon I)^{-1}(A+\varepsilon I)\right| a_{i}\right\rangle \leqq\left\langle a_{i}|(A+\varepsilon I)| a_{i}\right\rangle=a_{i}+\varepsilon$. Taking the limit $\varepsilon \rightarrow 0$ we obtain

$$
\left\langle a_{i}\left|A B^{-1} A\right| a_{i}\right\rangle \leqq\left\langle a_{i}|A| a_{i}\right\rangle .
$$

Hence $\operatorname{Tr}\left(A B^{-1} A\right)=\operatorname{Tr}\left(B^{-1} A^{2}\right) \leqq \operatorname{Tr} A$.

$A \leqq B$ implies that $\left\langle a_{i} \mid b_{j}\right\rangle=0$, if $a_{i} \neq 0$ and $b_{j}=0$. For $a_{i} \neq 0$ we have

$$
\begin{aligned}
& \left\langle a_{i}\left|\left(A \log A-A \log B-B^{-1} A^{2}+A\right)\right| a_{i}\right\rangle \\
& \quad=\sum_{b_{j} \neq 0}\left|\left\langle a_{i} \mid b_{j}\right\rangle\right|^{2} a_{i}\left[\log \left(a_{i} / b_{j}\right)-a_{i} / b_{j}+1\right] \leqq 0
\end{aligned}
$$

since $\log x \leqq x-1$ for $x>0$, with equality only for $x=1$.

As $\left\langle a_{i}|(A \log A-A \log B)| a_{i}\right\rangle=0$ for $a_{i}=0$, we get

$$
\operatorname{Tr}(A \log A-A \log B) \leqq \operatorname{Tr}\left(B^{-1} A^{2}-A\right) .
$$

Equality holds iff $\left\langle a_{i} \mid b_{j}\right\rangle \neq 0 \Rightarrow a_{i}=0$ or $a_{i}=b_{j}$. 
This condition is equivalent to

$$
B\left|a_{i}\right\rangle=\sum_{j}\left\langle b_{j} \mid a_{i}\right\rangle b_{j}\left|b_{j}\right\rangle=a_{i}\left|a_{i}\right\rangle=A\left|a_{i}\right\rangle
$$

for all $a_{i} \neq 0$. But Range $A$ is spanned by $\left\{\left|a_{i}\right\rangle ; a_{i} \neq 0\right\}$ so we get

$$
B \mid \text { Range } A=A \mid \text { Range } A \text {. }
$$

If this condition is fulfilled then obviously $\operatorname{Tr}\left(B^{-1} A^{2}-A\right)=0$ and the lemma is proved.

Proof of Theorem 1. Substitute $A+B$ for $B$ in the lemma, then interchange $A$ and $B$ and add the two inequalities. Then, if $S(A)$ and $S(B)$ are finite

$$
S(A+B) \leqq S(A)+S(B)
$$

with equality iff $(A+B) \mid$ Range $A=A \mid$ Range $A$ and $(A+B) \mid$ Range $B$ $=B \mid$ Range $B$ which is equivalent to $A \cdot B=0$.

If $S(A)$ or $S(B)=\infty$ then $S(A+B)=\infty$ from the concavity property of the entropy ([2], p. 28).

Corollary. Let $A,\left\{A_{i}\right\}$ be positive trace class operators. If $A=\Sigma A_{i}$ then

$$
S(A) \leqq \Sigma S\left(A_{i}\right) .
$$

If $S(A)<\infty$ then the equality holds iff $A_{i} \cdot A_{j}=0$ for $i \neq j$.

Proof. Let the eigenvalues of $A^{(n)}=\sum_{i=1}^{n} A_{i}$ be $\left\{a_{k}^{(n)}\right\}$ arranged in decreasing order. Then by [6], Chapter 2 , Lemma 1.1. $a_{k}^{(n)} \leqq a_{k}^{\left(n^{\prime}\right)}$ for $n \leqq n^{\prime}$, all $k$. From $\operatorname{Tr}\left(A-A^{(n)}\right) \rightarrow 0$ and monotonity follows that the eigenvalues of $A$ are $a_{k}=\lim _{n} a_{k}^{(n)}$. Let $h(x)=-x \log x$. For $k$ sufficiently large $\left(a_{k}<e^{-1}\right)$ and $n \leqq n^{\prime}$ we have $h\left(a_{k}^{(n)}\right) \leqq h\left(a_{k}^{\left(n^{\prime}\right)}\right)$ and the convergence $h\left(a_{k}^{(n)}\right) \rightarrow h\left(a_{k}\right)$ is monotone, hence $\lim S\left(A^{(n)}\right)=\lim \Sigma h\left(a_{k}^{(n)}\right)=\Sigma h\left(a_{k}\right)=S(A)$. Then $S\left(A^{(n)}\right) \leqq \sum_{1}^{n} S\left(A_{i}\right)$ implies that $S(A) \leqq \sum_{1}^{\infty} S\left(A_{i}\right)$. If $S(A)=\sum_{1}^{\infty} S\left(A_{i}\right)<\infty$, then equality holds in $S(A) \leqq S\left(A_{j}\right)+S\left(\sum_{i \neq j} A_{i}\right) \leqq \Sigma S\left(A_{i}\right)$ and $A_{j} \cdot \sum_{i \neq j} A_{i}=0$, hence $A_{i} \cdot A_{j}=0$ for $i \neq j$.

Theorem 2. Let $W$ be a state with $S(W)<\infty, W^{\prime}$ and $W_{k}^{\prime}$ as defined above. Then

$$
\Sigma p_{k} S\left(W_{k}^{\prime}\right) \leqq S(W) .
$$

Equality holds if and only if $S\left(W_{k}^{\prime}\right)=S(W)$ for all $k$ with $p_{k} \neq 0$.

Proof. The statement is trivial if $W$ is pure: $S(W)=S\left(W_{k}^{\prime}\right)=0$ all $k$. Let $W=\Sigma w_{i} W_{i}, W_{i}$ pure, $W_{i} \cdot W_{j}=0$ for $i \neq j$ and put $s\left\{w_{i}\right\}=-\Sigma w_{i} \log w_{i}$. 
Then $S(W)=s\left\{w_{i}\right\}$ and

$$
W_{k}^{\prime}=p_{k}^{-1} P_{k} W P_{k}=\sum_{i} w_{i} p_{i, k} W_{i, k}^{\prime}
$$

where $p_{i, k}=p_{k}^{-1} \operatorname{Tr} P_{k} W_{i}$ and $W_{i, k}^{\prime}=\left(\operatorname{Tr} P_{k} W_{i}\right)^{-1} P_{k} W_{i} P_{k}$. By the corollary of Theorem 1, noting that all $W_{i, k}^{\prime}$ are pure

$$
\begin{aligned}
S\left(W_{k}^{\prime}\right) \leqq \sum_{i} S\left(w_{i} p_{i, k} W_{i, k}^{\prime}\right)= & \sum_{i} w_{i} p_{i, k} S\left(W_{i, k}^{\prime}\right)+s\left\{w_{i} p_{i, k}\right\}=s\left\{w_{i} p_{i, k}\right\} \\
\text { i.e. } & \sum_{k} p_{k} S\left(W_{k}^{\prime}\right) \leqq \sum_{k} p_{k} s\left\{w_{i} p_{i, k}\right\} .
\end{aligned}
$$

Use the fact that $s\{\}$ is a concave function and $\Sigma p_{k}=1, \Sigma P_{k}=I$ :

$\sum_{k} p_{k} s\left\{w_{i} p_{i, k}\right\} \leqq s\left\{\sum_{k} p_{k} w_{i} p_{i, k}\right\}=s\left\{w_{i} \sum_{k} \operatorname{Tr}\left(P_{k} W_{i}\right)\right\}=s\left\{w_{i}\right\}=S(W)$.

If $S\left(W_{k}^{\prime}\right)=S(W)$ for all $k$ such that $p_{k} \neq 0$ then the equality obviously holds. Conversely, if $\Sigma p_{k} S\left(W_{k}^{\prime}\right)=S(W)$, then equality holds in (2). This implies that for each $i, w_{i} p_{i, k}$ are equal for all $k$ such that $p_{k} \neq 0([5], \S 3.8)$ i.e. $w_{i} p_{i, k}=\alpha_{i}$, hence

$$
w_{i} \operatorname{Tr}\left(P_{k} W_{i}\right)=\alpha_{i} p_{k} .
$$

Summation over $k$ gives $\alpha_{i}=w_{i}$, i.e. $p_{i, k}=1$ and

$$
\operatorname{Tr}\left(P_{k} W_{i}\right)=\left\langle i\left|P_{k}\right| i\right\rangle=p_{k} .
$$

Furthermore we must have equality in (1):

$$
S\left(W_{k}^{\prime}\right)=\sum_{i} S\left(w_{i} p_{i, k} W_{i, k}^{\prime}\right)=\Sigma S\left(w_{i} W_{i, k}^{\prime}\right)=s\left\{w_{i}\right\}=S(W)
$$

and the theorem is proved.

Remark. Equality in (1) implies by Theorem 1

$$
W_{i, k}^{\prime} W_{j, k}^{\prime}=0 \text { for } i \neq j
$$

hence $\operatorname{Tr}\left(P_{k} W_{i} P_{k} W_{j}\right)=\left|\left\langle j\left|P_{k}\right| i\right\rangle\right|^{2}=0$ for $i \neq j$.

This and the condition $p_{i, k}=1$ can be summarized by

$$
\left\langle i\left|P_{k}\right| j\right\rangle=p_{k} \delta_{i j} \quad \text { or } \quad W_{i} P_{k} W_{j}=p_{k} \delta_{i j} W_{i} .
$$

Introducing $P_{W}=\Sigma W_{i}$ (the support of $W$ ) this condition reads

$$
P_{W} P_{k} P_{W}=p_{k} P_{W} \text { for all } k,
$$

which is an equivalent condition for equality in Theorem 2 .

This relation is obviously satisfied if $W$ is pure. If $P_{W}=I$ the condition implies that $P_{k}=I$ and $p_{k}=1$ for one $k$. If the observable commutes with $W$ then $P_{k} P_{W}=p_{k} P_{W}$, hence $P_{W} \leqq P_{k}$ and $p_{k}=1$ for one $k$. 
There are also nontrivial cases provided the dimension of $P_{W}$ is not larger than the dimension of $I-P_{W}$.

Acknowledgement. I wish to thank Professor B. Nagel for many enlightening discussions.

\section{References}

1. Neumann,J.von: Mathematical foundations of quantum mechanics. Princeton: University Press 1965.

2. Ruelle, D.: Statistical mechanics. New York: Benjamin 1969.

3. Groenewold, H.J.: Int. J. Theor. Phys. 4, 327 (1971).

4. Lanford, O.E., Robinson, D. W.: J. Math. Phys. 9, 1120 (1968).

5. Hardy, G.H., Littlewood,J.E., Polya, G.: Inequalities. Cambridge: University Press 1934.

6. Gohberg,I.C., Krein,M.G.: Introduction to the theory of linear nonselfadjoint operators. Providence: Am. Math. Soc. 1969.

G. Lindblad

The Royal Institute of Technology

Department of Theoretical Physics

Lindstedtsvägen 15

S-10044 Stockholm 70, Sweden 
